NASA/CR-1999-209121

ICASE Report No. 99-15

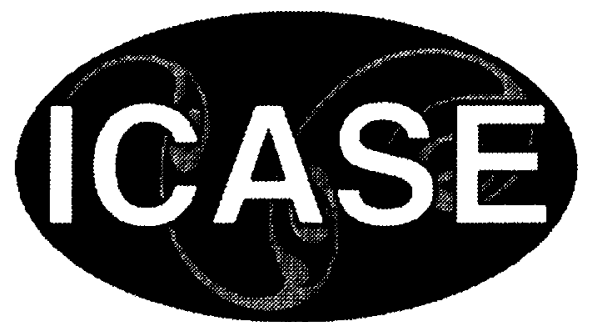

\title{
Reduction of Large Dynamical Systems by Minimization of Evolution Rate
}

Sharath S. Girimaji

ICASE, Hampton, Virginia

Institute for Computer Applications in Science and Engineering NASA Langley Research Center

Hampton, VA

Operated by Universities Space Research Association

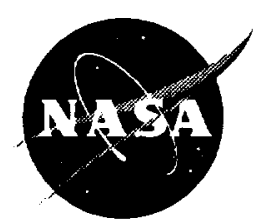

National Aeronautics and

Space Administration

Langley Research Center

Hampton, Virginia 23681-2199 
Available from the following:

NASA Center for AeroSpace Information (CASI) 7121 Standard Drive

Hanover, MD 21076-1320

(301) 621-0390

National Technical Information Service (NTIS)

5285 Port Royal Road

Springfield, VA 22161-2171

(703) 487-4650 


\title{
REDUCTION OF LARGE DYNAMICAL SYSTEMS BY MINIMIZATION OF EVOLUTION RATE
}

\author{
SHARATH S. GIRIMAJI*
}

\begin{abstract}
Reduction of a large system of equations to a lower-dimensional system of similar dynamics is investigated. For dynamical systems with disparate timescales, a criterion for determining redundant dimensions and a general reduction method based on the minimization of evolution rate are proposed.
\end{abstract}

Key words. deduced chemical kinetics, low-dimensional manifolds, algebraic stress modeling

Subject classification. Turbulent Combustion

1. Introduction. The macroscopic behavior of many complex systems (with a large number of degrees of freedom or scales) is largely insensitive to the details of the microscopic features. The macroscopic behavior can then be nearly exactly described by a simpler system with very few degrees of freedom or scales. In this article, a general method for reducing the description of the macroscopic behavior of large systems is presented. The two specific examples considered in this paper are of importance in the modeling and computation of turbulent combustion: the reduction of complex chemical kinetics and algebraic modeling of Reynolds stresses.

Consider an autonomous dissipative dynamical system with one attracting fixed point:

$$
\dot{\mathbf{z}}=\mathbf{g}(\mathbf{z}), \text { where } \mathbf{z}=\left(z_{1}, z_{2}, \cdots z_{n}\right)
$$

It is assumed that $\mathbf{z}$ is suitably scaled and nondimesionalized. If the system has disparate timescales, the solution exhibits a typical three-stage behavior. (i) Initial-condition dependent initial transient stage which lasts until all the small-timescale fast processes are exhausted; (ii) the intermediate slow-manifold stage in which the solutions 'bunch' together in a lower-dimensional phase space as the slow processes dominate; and (iii) the final equilibrium state. In the slow-manifold stage, the degrees of freedom of the system can be reduced if the relationship between fast and slow variables can be found. In a nonlinear dynamical system it is difficult to characterize the slow manifold accurately and the current practice is to locally linearize the equations. We will use the example of a general two-variable linear system with one large $(-1)$ and one small $(-\varepsilon)$ eigenvalue in our analysis:

$$
\begin{aligned}
& \dot{x}=-x\left(\cos ^{2} \theta+\varepsilon \sin ^{2} \theta\right)+y \sin \theta \cos \theta(1-\varepsilon) \\
& \dot{y}=x \sin \theta \cos \theta(1-\varepsilon)-y\left(\sin ^{2} \theta+\varepsilon \cos ^{2} \theta\right)
\end{aligned}
$$

The directions corresponding to the two eigenvalues are

$$
x=-y \cot \theta \text { for } \lambda=-1 ; x=+y \tan \theta \text { for } \lambda=-\varepsilon
$$

In a linear system, the slow manifold lies in a space spanned by the eigenvectors corresponding to small (in magnitude) eigenvalues. Maas and Pope [1] determine the slow-manifold by requiring it to be orthogonal

*Institute for Computer Applications in Science and Engineering (ICASE), NASA Langley Research Center, Hampton, VA 23681-2199. This research was supported by the National Aeronautics and Space Administration while the author was in residence at the Institute for Computer Applications in Science and Engineering (ICASE), NASA Langley Research Center, Hampton, VA 23681-2199. 
to the principal directions corresponding to the large (negative) eigenvalues of the Jacobian (MP model). In the example considered the slow-manifold is

$$
x(\text { slow-manifold })=+y \tan \theta .
$$

The determination of the eigenvalues and eigen-directions of the Jacobian and subsequently the slow manifold can be prohibitively expensive in a practical nonlinear problem involving several dozen variables. The popular and inexpensive steady-state approximation (SSA) method of reduction involves setting the rate of change of fast variables to zero to obtain algebraic relations between fast and slow variables. In the sample problem,

$$
\begin{aligned}
& x(\text { slow-manifold })=+y \tan \theta\left(1-\varepsilon \sec ^{2} \theta+\varepsilon^{2} \sec ^{2} \theta \tan ^{2} \theta+O\left(\varepsilon^{3}\right)\right), \quad \text { if } \mathrm{x} \text { is fast variable, } \\
& y(\text { slow-manifold })=+x \cot \theta\left(1-\varepsilon \csc ^{2} \theta+\varepsilon^{2} \csc ^{2} \theta \cot ^{2} \theta+O\left(\varepsilon^{3}\right)\right), \quad \text { if } \mathrm{y} \text { is fast variable. }
\end{aligned}
$$

Judicious choice of the steady state variable is crucial and this is usually made from a priori knowledge. Even with a proper choice, the error incurred is first order in the eigenvalue ratio. For further details about these methods and other important developments the reader is referred to [2], [3] and [4].

Our objectives are to (i) devise a general criterion for selecting fast variables and (ii) develop a computationally viable reduction procedure of higher (than one) order accuracy in eigenvalue ratio.

2. Characterization of Slow Variables. Fast variables are those whose evolution is dominated by the large eigenvalues of the Jacobian and the slow variables by the small eigenvalues. In fact, close to the slow-manifold, fast variable evolution can be slower than that of slow variables rendering evolution rate an unsuitable selection criterion. A better gauge of the timescale of a variable $\left(z_{i}\right)$ is the convergence rate of its value between two neighboring trajectories. The difference in $z_{i}$ values between two neighboring trajectories, $\delta z_{i}$, evolves according to

$$
\frac{d\left(\delta z_{i}\right)}{d t}=\sum_{j=1}^{n} \frac{\partial g_{i}}{\partial z_{j}}\left(\delta z_{j}\right)
$$

Based on this, two approximate measures of the dissipativeness of individual variables are proposed for the selection criterion:

$$
D_{i}=\frac{\partial g_{i}}{\partial z_{i}} ; \text { or } D_{i}=\left[\sum_{j=1}^{n}\left(\frac{\partial g_{i}}{\partial z_{j}}\right)^{2}\right]^{\frac{1}{2}}
$$

In general, large magnitude of $D_{i}$ implies fast variable and slow variables are characterized by small magnitudes. When the physical and eigen variables coincide, it is easy to see that $D_{i}$ are indeed the eigenvalues. While the second estimate is likely to be more accurate in general, the first measure may be adequate when the off-diagonal terms in the Jacobian are relatively small.

3. Reduction of the Dynamical System. Each state in phase-space is associated with a residence timescale which is proportional to the amount of time a solution trajectory resides in an infinitesimal neighborhood of that point and inversely proportional to the local evolution rate $\left(V=\sqrt{\sum_{i=1}^{n} g_{i} g_{i}}\right)$. Solutions tend to stagnate near long-timescale states and pass quickly through short-timescale states. The probability of finding a solution, of unknown time lapse and arbitrary initial condition, at a given state along its trajectory is proportional to residence timescale of that state. This leads to the premise of our reduction procedure. Given the values of slow variables, an arbitrary solution trajectory is most likely to be found at the state with the longest residence time, i.e., smallest evolution rate. This proposal is as valid for non-linear systems as 
it is for linear systems and bears semblance to the maximum likelihood estimator of mathematical statistics and the non-equilibrium potential concept of [5]. When not conditioned by any slow-variable values, this criterion selects the equilibrium state as the most likely state of the system. If we represent the retained (slow) variables in $\mathbf{z}$ by the vector $\mathbf{y}$ and the discarded (fast) variables by the vector $\mathbf{x}$, the statement of our proposal can be written as:

$$
\mathbf{x}(\mathbf{y}) \approx \min _{\mathbf{x}} F(\mathbf{x}: \mathbf{y}), \quad \text { where } F=V^{2}=\sum_{i=1}^{n} g_{i} g_{i}
$$

In the present example

$$
\begin{aligned}
& F(x, y)=\left[\frac{d x}{d t}\right]^{2}+\left[\frac{d y}{d t}\right]^{2} \\
= & x^{2}\left(\cos ^{2} \theta+\varepsilon^{2} \sin ^{2} \theta\right)+y^{2}\left(\sin ^{2} \theta+\varepsilon^{2} \cos ^{2} \theta\right)-2 x y \sin \theta \cos \theta\left(1-\varepsilon^{2}\right) .
\end{aligned}
$$

The value of $x$ that minimizes the evolution rate is

$$
\begin{aligned}
x(y) & =y \frac{\sin \theta \cos \theta\left(1-\varepsilon^{2}\right)}{\cos ^{2} \theta+\varepsilon^{2} \sin ^{2} \theta} \\
& =y \tan \theta\left[1-\left(1+\tan ^{2} \theta\right) \varepsilon^{2}+O\left(\varepsilon^{4}\right)\right] .
\end{aligned}
$$

When the eigen and physical variables coincide, there is no error involved; otherwise, it is of order $\varepsilon^{2}$ which makes this method more accurate than the SSA method. The fast variable selection criterion given above will ensure that $\theta \leq \pi / 4$.

For even better accuracy, minimization of the evolution rates of higher-order derivatives of the variables is proposed. Differentiation has the effect of separating the timescales so that the timescales of $\dot{\mathbf{x}}$ and $\dot{\mathbf{y}}$ are farther apart than the those $\mathbf{x}$ and $\mathbf{y}$. The higher the order of the derivative, the greater is the separation between the slow and fast variable (derivatives). In matrix algebra, this fact is the basis of the power method of separating small and large eigenvalues. Our proposal for higher-order accuracy is

$$
\mathbf{x}(\mathbf{y}) \approx \min _{\mathbf{x}} F^{m}(\mathbf{x}: \mathbf{y}) ; \quad \text { where } F^{m}(\mathbf{z})=\sum_{i=1}^{n} \frac{d^{m} z_{i}}{d t^{m}} \frac{d^{m} z_{i}}{d t^{m}}
$$

In our sample problem,

$$
\begin{aligned}
F^{m}(x, y)= & x^{2}\left(\cos ^{2} \theta+\varepsilon^{2 m} \sin ^{2} \theta\right)+y^{2}\left(\sin ^{2} \theta+\varepsilon^{2 m} \cos ^{2} \theta\right) \\
& -2 x y \sin \theta \cos \theta\left(1-\varepsilon^{2 m}\right)
\end{aligned}
$$

which leads to

$$
x(y)=y \tan \theta\left[1-\left(1+\tan ^{2} \theta\right) \varepsilon^{2 m}+O\left(\varepsilon^{4 m}\right)\right] .
$$

In practical problems, minimization of $F^{1}$ may be adequate as the error involved in local linearization may be of order $\varepsilon^{2}$. More than one minima of the evolution rate may be encountered in the proximity of other (unstable) fixed points. (This will also be the case with SSA method.) Then, the minimum that has all negative eigenvalues (attractor) must be chosen. The computational effort is still much smaller than the MP method, where eigenvalues and eigenvectors are evaluated at all points in the fast phase-space as opposed to eigenvalues only at a handful of minima in the present method. 
4. Chemical Kinetics Reduction. The phenomenon of turbulent combustion encompasses a wide spectrum of length and time scales. Typically, the thermo-chemical timescales representing chemical reactions span a wider range than those of turbulent advection and molecular diffusion. In the interest of computational efficiency, it is desirable to eliminate chemical reactions and species whose characteristic timescales are smaller than the fluid timescales.
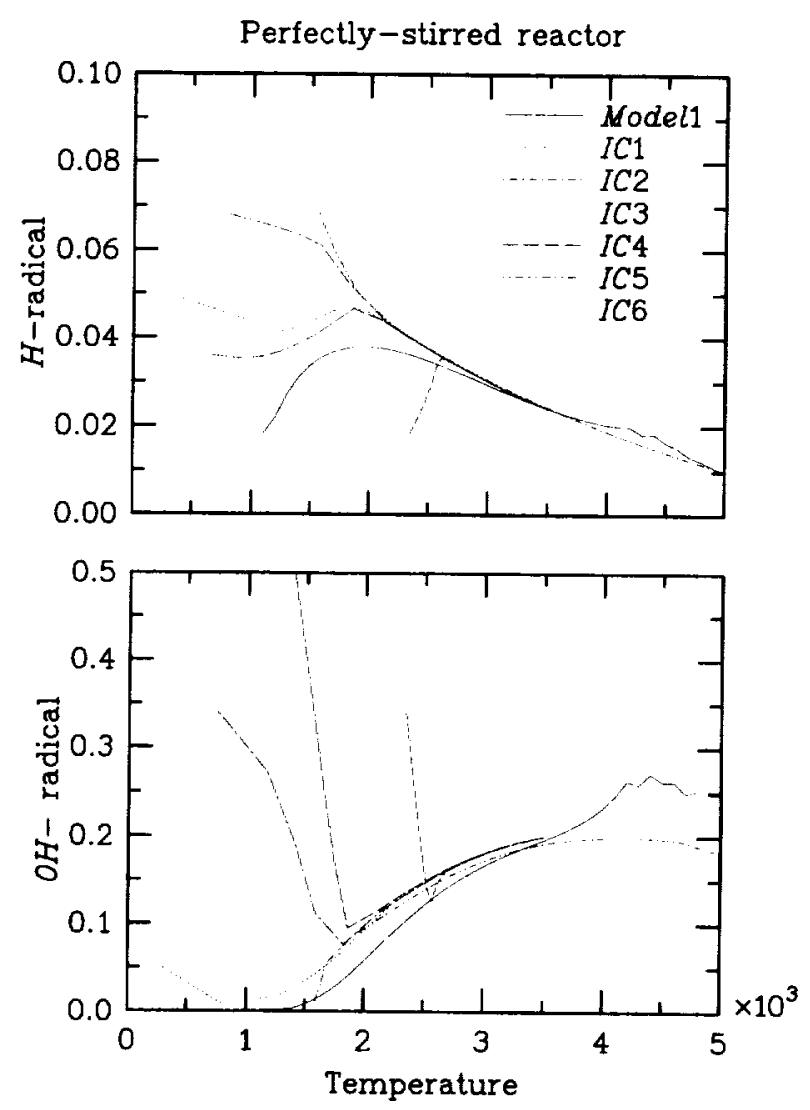

FIG. 4.1. State-space evolution of $H$ and $O H$ as a function of temperature. Different dashed and dotted lines correspond to results from full kinetics simulations from different initial conditions. The solid line represents the slow-manifold model prediction with temperature as the only retained variable.

Consider chemical reactions among $N_{s}$ chemical species involving $N_{r}$ reactions in an adiabatic, isobaric, well-stirred reactor. The species (mass fraction $Y_{i}$ ) evolve according to

$$
\frac{\partial Y_{i}}{\partial t}=R_{i}=W_{i} \sum_{j=1}^{N_{R}} \frac{\beta_{i j}}{\rho^{\alpha_{j}}} A_{j} T^{b_{j}} \exp \left(-\frac{E_{j}}{\mathcal{R} T}\right) \prod_{n=1}^{N_{S}}\left(\frac{Y_{n}}{W_{n}}\right)^{\beta_{n j}}
$$

where: $W_{i}$ is the molecular weight of species $i ; \beta_{i j}$ is the stoichiometric coefficient of species $i$ in the $j$-th reaction; $\alpha_{j}=\sum_{i=1}^{N_{a}} \beta_{i j} ; A_{j}, b_{j}$ and $E_{j}$ are the Arrehenius constants; and, $\mathcal{R}$ is the universal gas constant. Temperature and density are obtained from

$$
\begin{aligned}
C_{p} \frac{d T}{d t} & =-\sum_{i=1}^{N_{S}} \Delta h_{i, 0}\left[\frac{d Y_{i}}{d t}\right], \\
\rho\left(p, T, Y_{i}\right) & =\frac{p}{\mathcal{R} T}\left(\sum_{i=1}^{N_{S}} \frac{Y_{i}}{W_{i}}\right)^{-1} .
\end{aligned}
$$


We consider the evolution of a $\mathrm{H}_{2} / \mathrm{O}_{2}$ mixture, in which the atomic mass fractions of hydrogen and oxygen are 0.15 and 0.85 respectively, from various initial conditions. The constant total enthalpy is $1.104 \times 10^{7}$ $\mathrm{KJ}$ and the constant pressure is $2 \mathrm{~atm}$. The detailed 14-step chemical mechanism involves temperature $(T)$ and six species $\left(\mathrm{O}_{2}, \mathrm{O}, \mathrm{H}_{2}, \mathrm{H}, \mathrm{OH}\right.$, and $\left.\mathrm{H}_{2} \mathrm{O}\right)$. The equilibrium state is given by: $T=3506 \mathrm{~K} ; Y_{\mathrm{H}}=0.024$; $Y_{\mathrm{H}_{2} \mathrm{O}}=0.556 ; Y_{\mathrm{OH}}=0.201 ; Y_{\mathrm{O}}=0.086 ; Y_{\mathrm{H}_{2}}=0.060$ and $Y_{\mathrm{O}_{2}}=0.070$.
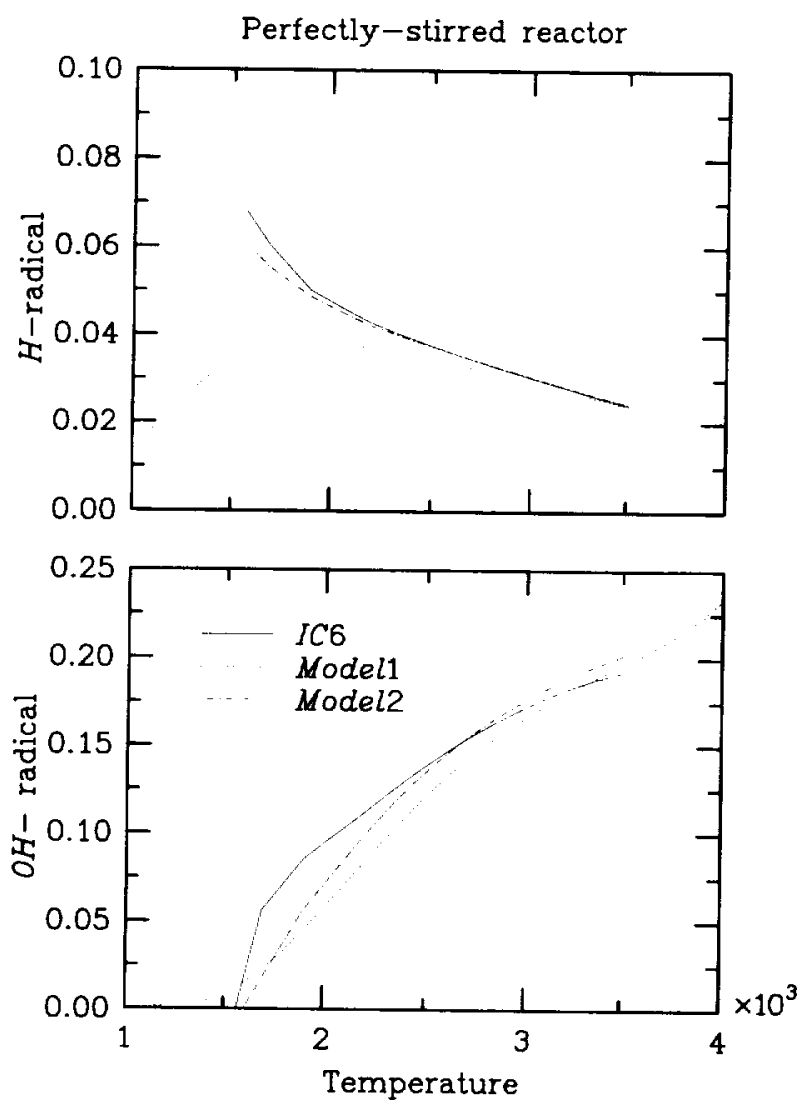

FIG. 4.2. State-space evolution of $H$ and $O H$ as a function of temperature. Solid line - case IC5 urith full kinetics; dotted line - reduced model with temperature as the only retained variable; dot-dash line - reduced model with temperature and $\mathrm{H}_{2} \mathrm{O}$ as the retained variables.

Evolution of the species undergoing reactions according to the detailed chemical kinetics from six arbitrary initial conditions is calculated numerically using a second-order Runge-Kutta procedure. The evolution trajectories of $\mathrm{H}$ and $\mathrm{OH}$ are shown in Figure 4.1. A one-dimensional slow manifold model is constructed with temperature as the only retained variable:

$$
\mathbf{Y}(T) \approx \min _{\mathbf{Y}}\left[\sum_{i=1}^{N_{s}} R_{i} R_{i}+\frac{d T}{d t} \frac{d T}{d t}\right]
$$

The mass fractions of $\mathrm{H}$ and $\mathrm{OH}$ predicted by the one-dimensional model is also shown in Figure 4.1. The agreement is quite adequate. Next we construct a two-dimensional manifold with water mass fraction as a retained variable along with temperature. Comparison between the two-dimensional model and detailed kinetics is made for the case IC5 in Figure 4.2. The one-dimensional model values are also shown for comparison. The agreement between the two-dimensional model and detailed kinetics is, in general, excellent. These results demonstrate that the present method is an accurate and computationally viable option for 
reducing chemical kinetics.

5. Algebraic Reynolds Stress Modeling. In the field of turbulence modeling, closure of Reynolds stress transport equations is the lowest level of sophistication at which models can be developed in a systematic manner from the governing Navier-Stokes equations. This entails solving seven modeled transport equations for turbulence variables - five for Reynolds stress anisotropy components $b_{i j}$ and one each for turbulent kinetic energy $K$ and dissipation $\varepsilon-$

$$
\begin{aligned}
\frac{d b_{i j}}{d t}= & -b_{i j}\left(L_{1}^{0} \frac{\varepsilon}{K}-L_{1}^{1} b_{m n} S_{m n}\right) \\
& +L_{2} S_{i j}+L_{3}\left(b_{i k} S_{j k}+b_{j k} S_{i k}-\frac{2}{3} b_{l m} S_{l m} \delta_{i j}\right) \\
& +L_{4}\left(b_{i k} W_{j k}+b_{j k} W_{i k}\right) \\
\frac{d K}{d t}= & -K b_{i j} S_{i j}-\varepsilon \\
\frac{d \varepsilon}{d t}= & -C_{e 1} \varepsilon b_{i j} S_{i j}-C_{e 2} \frac{\varepsilon^{2}}{K} .
\end{aligned}
$$

The Einstein summation convention is used. In the above equations $L$ 's and $C$ 's are model coefficients, and $S_{i j}$ and $W_{i j}$ are the mean flow strain and rotation rates. For the sake of brevity, the coefficient values used are not given here and the reader is referred to [6].

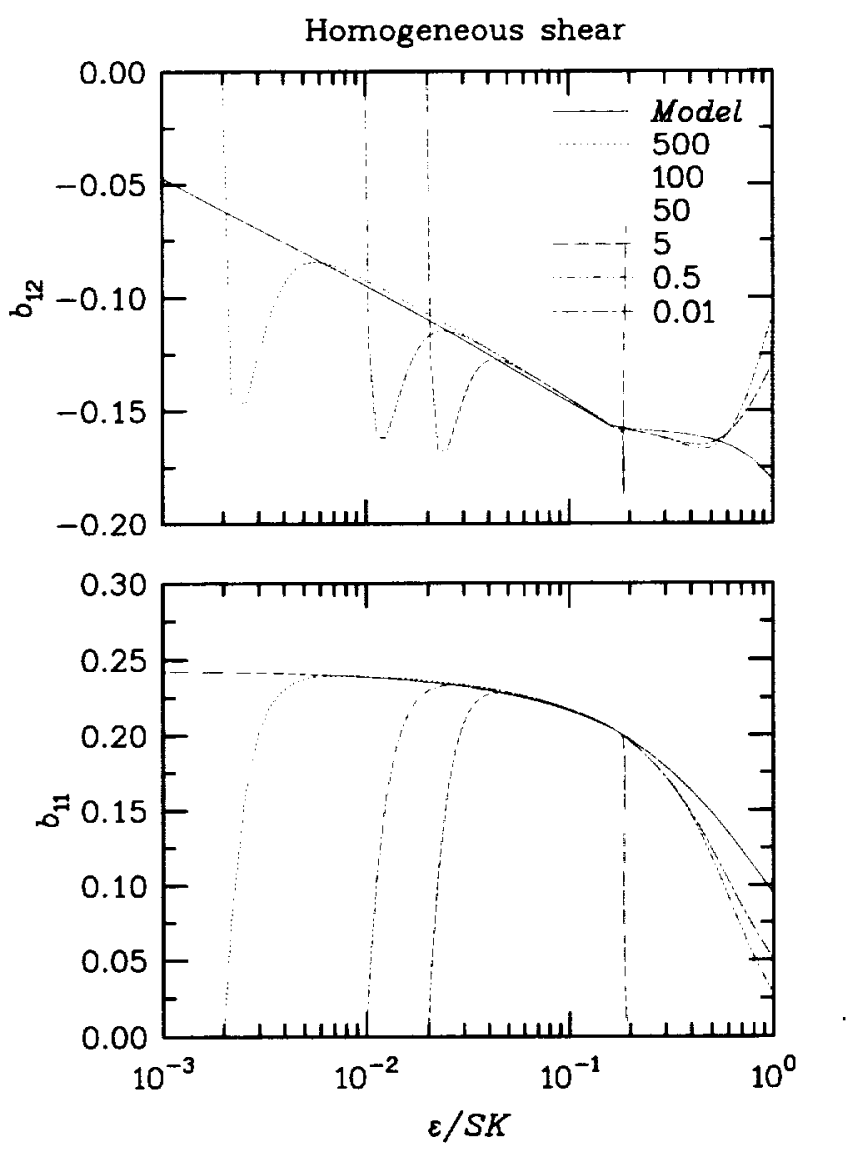

FIG. 5.1. Homogeneous turbulence. Evolution of $b_{12}$ and $b_{11}$ as a function of $w$. Solid line - reduced model with $K$ and $\varepsilon$ as the retained variables; dashed and dotted lines - full Reynolds stress model calculations from various initial conditions. 
Researchers have long sought to reduce the seven-equation model to a computationally affordable twoequation (for $K$ and $\varepsilon$ ) model. The Reynolds stress anisotropies are the discarded variables for which we seek algebraic expressions: hence the name, algebraic stress model. The evolution rate of this system in state-space is given by,

$$
F=V^{2}=\frac{d b_{i j}}{d t} \frac{d b_{i j}}{d t}+\frac{d K}{d t} \frac{d K}{d t}+\frac{d \varepsilon}{d t} \frac{d \varepsilon}{d t} .
$$

The algebraic stress model assumption for non-equilibrium turbulent flows is

$$
\mathbf{b}(K, \varepsilon)=\min _{\mathbf{b}} F(\mathbf{b}: K, \varepsilon) .
$$

For two-dimensional mean flows, the minimization is performed in a three-dimensional discarded variable state space of $b_{11}, b_{12}$ and $b_{33}$.

We test the model in homogeneous shear turbulence in which the only non-zcro values of mean strain and rotation tensor components are

$$
S_{12}=S_{21}=W_{12}=-W_{21}=\frac{S}{2},
$$

where $S$ is a constant. The seven-equation model calculations for various $S$ values are shown in Figure 5.1. Each anisotropy is plotted as a function of the self-similarity (retained) variable $\varepsilon / S K$. The equilibrium values are: $\omega=0.166 ; b_{11}=0.204 ; b_{12}=-0.157 ; b_{22}=-0.149 ;$ and $b_{33}=-0.055$. The various solution trajectories exhibit a well-defined slow manifold behavior. The algebraic model, also shown in the figure, does an excellent job of reproducing the behavior of the full set. This algebraic model has been tested in a variety of other homogeneous flows with good success [7].

6. Conclusion. In summary, we have developed a general procedure for reducing dimensionality of autonomous systems with disparate timescales. The premise of the proposal is that the solution trajectories from unknown initial conditions are most likely to be found near the 'bottle-necks' in state-space which are characterized by small evolution rates. The location of these bottle-necks on the path to equilibrium can be found by simply minimizing the evolution velocity subject to constraints (given values of the slow or retained variables).

Acknowledgements. This work was performed while the author was a visiting scientist at Aeronautical Engineering Department of Indian Institute of Sciences, Bangalore, India. Assistance received from Professors K. N. Lakshmisha and H. S. Mukunda is gratefully acknowledged.

\section{REFERENCES}

[1] U. MaAs ANd S.B. Pope, Simplifying Chemical Kinetics: Intrinsic Low-dimensional Manifolds in Composition Space, Combustion and Flame 88 (1992), pp. $239-264$.

[2] H. HAKen, Synergetics: An Introduction, Springer-Verlag, 1977.

[3] N. PETERs, in Reduced kinetic mechanisms and asymptotic approximations for methane-air flames, M.D. Smooke, ed., Springer-Verlag, 1991.

[4] S.H. LAM AND D.A. Goussis, in Proceedings of 22nd Symposium (International) on Combustion, pp. 931-941, The Combustion Institute, Pittsburgh, 1988.

[5] T. Tel, R. Graham and G. Hu, Non-equilibrium potentials and their power series expansions, Phys. Rev. A 40 (1989), p. 4065. 
[6] S.S. GirimajI, Pressure-strain correlation modeling of complex turbulent flows, submitted to the J. Fluid Mech. (1999).

[7] S.S. GirimaJI, in Proceedings of Modeling Complex Turbulent Flows Symposium, Salas, Hefner and Sakell, eds., Kluwer Academic Publishers, 1998. 


\section{REPORT DOCUMENTATION PAGE}

Public reporting burden for this collection of information is estimated to average 1 hour per response. including the time for reviewing instructions, searching existing data sources. gathering and maintaining the data needed, and completing and reviewing the collection of information. Send comments regarding this burden estimate or any other aspect of this collection of information, including suggestions for reducing this burden, to Washington Headquarters Services, Directorate

\begin{tabular}{|c|c|c|}
\hline 1. AGENCY USE ONLY(Leave blank) & $\begin{array}{l}\text { 2. REPORT DATE } \\
\text { May } 1999\end{array}$ & $\begin{array}{l}\text { 3. REPORT TYPE AND } \\
\text { Contractor Report }\end{array}$ \\
\hline
\end{tabular}

4. TITLE AND SUBTITLE

Reduction of Large Dynamical Systems by Minimization of Evolution

Rate

5. FUNDING NUMBERS

C NAS1-97046

WU 505-90-52-01

6. AUTHOR(S)

Sharath S. Girimaji

\section{PERFORMING ORGANIZATION NAME(S) AND ADDRESS(ES)}

Institute for Computer Applications in Science and Engineering

Mail Stop 132C, NASA Langley Research Center

Hampton, VA 23681-2199

9. SPONSORING/MONITORING AGENCY NAME(S) AND ADDRESS(ES)

National Aeronautics and Space Administration

Langley Research Center

Hampton, VA 23681-2199
8. PERFORMING ORGANIZATION REPORT NUMBER

ICASE Report No. 99-15

10. SPONSORING/MONITORING AGENCY REPORT NUMBER

NASA/CR-1999-209121

ICASE Report No. 99-15

\section{SUPPLEMENTARY NOTES}

Langley Technical Monitor: Dennis M. Bushnell

Final Report

To appear in Physical Review Letters

12a. DISTRIBUTION/AVAILABILITY STATEMENT

12b. DISTRIBUTION CODE

Unclassified-Unlimited

Subject Category 34

Distribution: Nonstandard

Availability: NASA-CASI (301) 621-0390

13. ABSTRACT (Maximum 200 mords)

Reduction of a large system of equations to a lower-dimensional system of similar dynamics is investigated. For dynamical systems with disparate timescales, a criterion for determining redundant dimensions and a general reduction method based on the minimization of evolution rate are proposed.

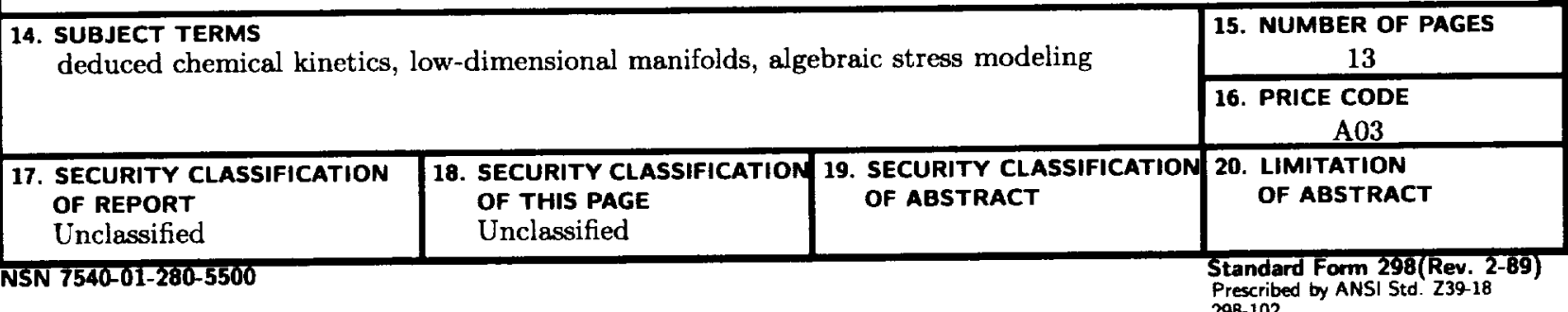


